DATA COMMUNICATION VIA BLUETOOTH BETWEEN PENDRIVES USING ARM

\author{
Abhijeet Ashish*1, Gaurav Gautam², Arjun Sahi ${ }^{3}$ \\ *11abhijeet.17sep@gmail.com, ${ }^{2}$ gauravgautam94@gmail.com, ${ }^{3}$ ansh.sahi@gmail.com
}

*Corresponding Author: -

Email: abhijeet.17sep@gmail.com

\begin{abstract}
: -
Now a days pendrive become so indespensible thing in our life. Any type of data is use share we need pendrive, to transfer those data first off all we need to put those datas in PC then into pendriveitissohecticprocess sotoovercomefromthislong process weneedapendrivewith inbuilt bluetooth. By this pendrive we can share data directly from pendrive to pendrive without need of a PC. This is very effective process which can save time as well as effort of user.It will make user comfortable to share data eaisly. to work with thispen drive we need a smallpowersupply tothependrivealmost 9 -rolt DCpower supply. Itcanbedual meansitcan trasfer data usually with the help of PCorvia bluetooth when power supplyiven to it.
\end{abstract}

Key words: Pendrive, USB, Bluetooth, PC, Laptops, Battery, Data, ARM, USB controller IC VNCIL, USB FLASH, NAND flash memory, crystal ocillator, hardware, software.

\title{
(a) $(\$)$
}




\section{INTRODUCTION:}

Now adaysitisessential to share dataas perourneedfortransferring thedatasweneedusb orpendriveormobilephone.Basically itisseenpendriveisusuallyseentouse.Manydatasand applications are developed daily which common computer user has to transfer from one USB Flash device into another, with the minimum wastage of time. For this user has to first find a computer then wait for it to boot up, then plug in his device, and then transfer the data. Different types of USB ash devices are used now-a-days. It is not necessary that all of these devices are supported by the computer and the operating system and their device drivers are available and installed. Carrying a computer or a laptop just for the sake of data transfer is not affordable these days in the age when people want all devices to be handy. More- over, transferring data via a computer involves a lot of power to be wasted, since the computer has to be entirely functional before it can transfer data. Also, the threat of viruses and malware has made the life of computer users more complicated. These viruses get activated as soon as the device is plugged into the system and get copied along with other data from one ash device into another. Our project here can provide a valuable solution to all problems faced by person in above situations. Our aim is to build a small and handy device to transfer data from one USB Flash device to another. Carrying a computer or a laptop just for the sake of data transfer is not affordable these days in the age when people used to carry a very light wait so usually it is seen that bluetooth in pendrive should be came into exisistance. To make this system we need following things:

a. Flash Drive USB 2.0 with Bluetooth chip

b. $9 \mathrm{~V}$ battery

c. Hardware to install Bluetooth drivers

d. Turn on/off switch for Bluetooth

\section{Overview of Project:}

In this project we need a flesh drive of $1 \mathrm{~GB}$ which is used to transmit and recieve data between itself and other device without help of any wire. In this project we may use infrared and satellite communication here insted of bluetooth but due to short range of them we have to use bluetooth here.

\section{Universal Serial Bus (USB)}

USB was designed to be an interface for communicating with many types of peripherals without the Limits and frustrations of older interfaces. Every recent PC includes USB ports that can connect to standard peripherals such as keyboards, mice, scanners, cameras, printers, and storage drives. It is a very useful protocol designed for a computer to communicate with almost any type of peripheral. Some of its benefits for users include:

1. Single Interface for many devices.

2. Considerably high Data rate.

3. Automatic Configuration.

4. Easy connection.

5. Hot Pluggable.

6. No user Settings.

7. Frees Hardware Resources for other devices

\section{Bluetooth:}

Bluetooth is a techonology in which we can share datas without help of any wire. Its range is short allmost in the ISM band 2400-2480 MHz.

\section{Use of Bluetooth:}

It ia radio technology called frequency-hopping spread spectrum, which chops up the data being sent and transmits chunks of it on up to 79 bands ( $1 \mathrm{MHz}$ each; centered from 2402 to $2480 \mathrm{MHz}$ ) in the range 2,400-2,483.5 MHz (allowing for guard bands).

File transfer speeds vary considerably. Speeds may be given in Megabyte per second, Mbit per second or optical drive multipliers such as "180X" (180 times $150 \mathrm{KiB}$ per second). Typical fast drives claim to read at up to 30 megabytes/s $(\mathrm{MB} / \mathrm{s})$ and write at about half that speed. This is about 20 times faster than USB 1.1 "full speed" devices, which are limited to a maximum speed of $12 \mathrm{Mbit} / \mathrm{s}(1.5 \mathrm{MB} / \mathrm{s})$.

Essential Components: There are typically four parts to a flash drive:

a. Standard-A USB plug-provides a physical interface to the host computer.

b. USB mass storage controller- a small microcontroller with a small amount of on-chip ROM and RAM.

c. NAND flash memory chip(s) - stores data (NAND flash is typically also used in digital cameras).

d. Crystal oscillator- produces the device's main $12 \mathrm{MHz}$ clock signal and controls the device's data output through a phase-locked loop 
1. USB Standard, Male A-Plug.

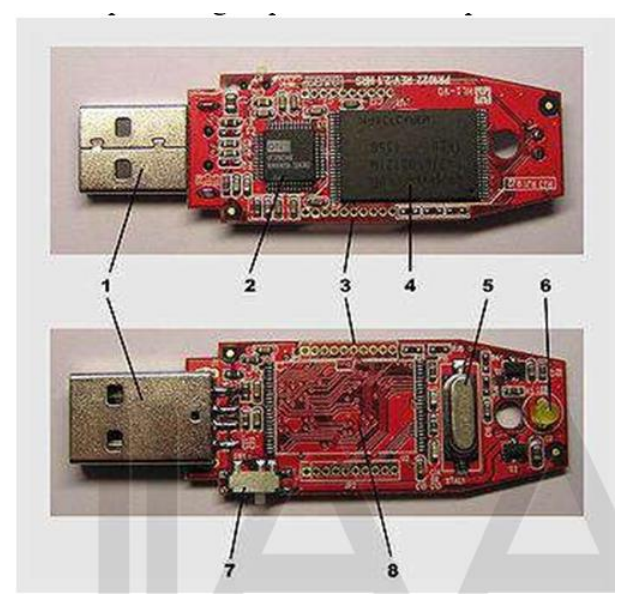

2. USB mass storage controller device/

3. Test Point.

4. Flash Memory Chip.

5. Crystal Oscillator.

6. LED (Optional).

7. Write-Protect switch (Optional)

8. Space for second Flash Memory Chip.

\section{Working block diagram:}

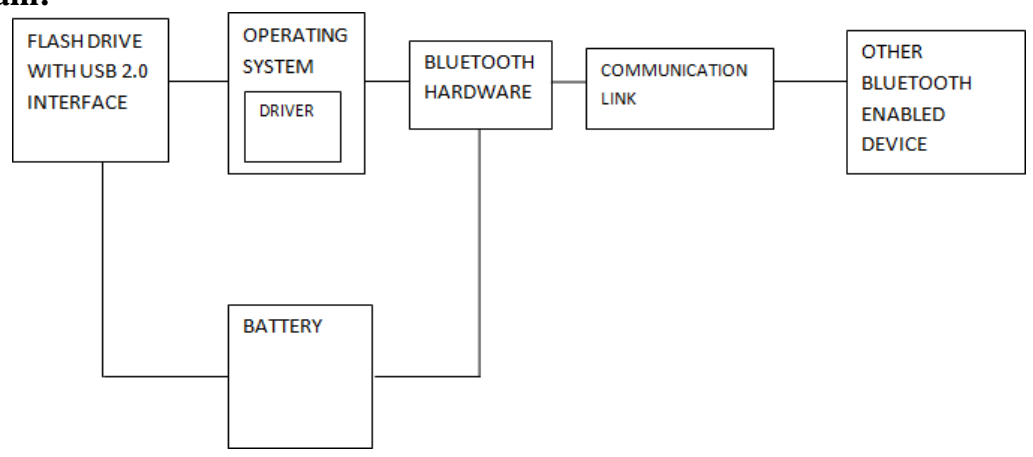

a. Flash Drive with USB interface: It is the pen drive for storing the data. They are also called as flash drive.

b. Operating System: It is used so that the drivers of Bluetooth and file manager can be installed in it using the operating system.

c. Bluetooth Hardware: It is the Bluetooth chip or device used to create a link between two or more operating devices. Here WT12 Bluetooth chip will be used manufactured by BLUEGIGA Pvt Ltd.

d. Communication Link: It is the link through which data will travel from other Bluetooth enabled devices to the pen drive or vice versa.

e. Battery: It is used to provide the power to Bluetooth chip and memory drive so that it can perform read/write operation.

f. Driver: It is required to allow the use of Bluetooth over the processor used and also for some data handling process.

\section{Implementation Detail:}

System block diagram

The study of the block diagram is very helpful to the designer. In this system we use ARM.

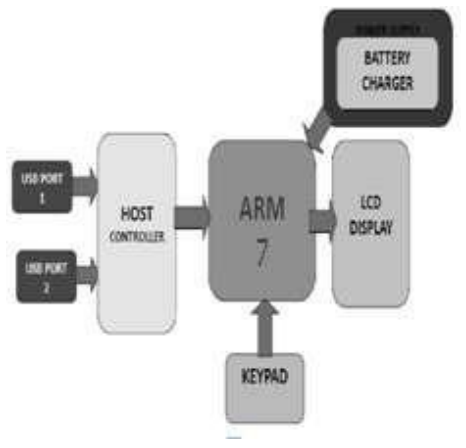

In this block diagram we clearly see that two USB port provide input fot host controller and another input is given by keypad then output displays on LCD display. Battery provides power supply. 
ARM:

ARM (LPC 2138): It is the heart of the system. It is prominent unit of the system because it reads data which we programmed and stored into its ROM, Then it executes it and controls the Display as well as Vinculum VNC1L Embedded USB Host controller. When key is press it read the data from source pen drive and writes to destination pen drive.

\section{USB Controller IC VNC1L:}

The VNC1L is the first generation device of FTDI Vinculum family of USB host/slavecontroller ICs, which are designed to deliver USB host level interfacing capablities to embedded application. The VNC1L features two independent USB host/slave with an inbuilt 8/32- bit MCU proccessing core with 4kBytes of RAM program memory and 64kBytes of Embedded flash memory for firmeare storage.

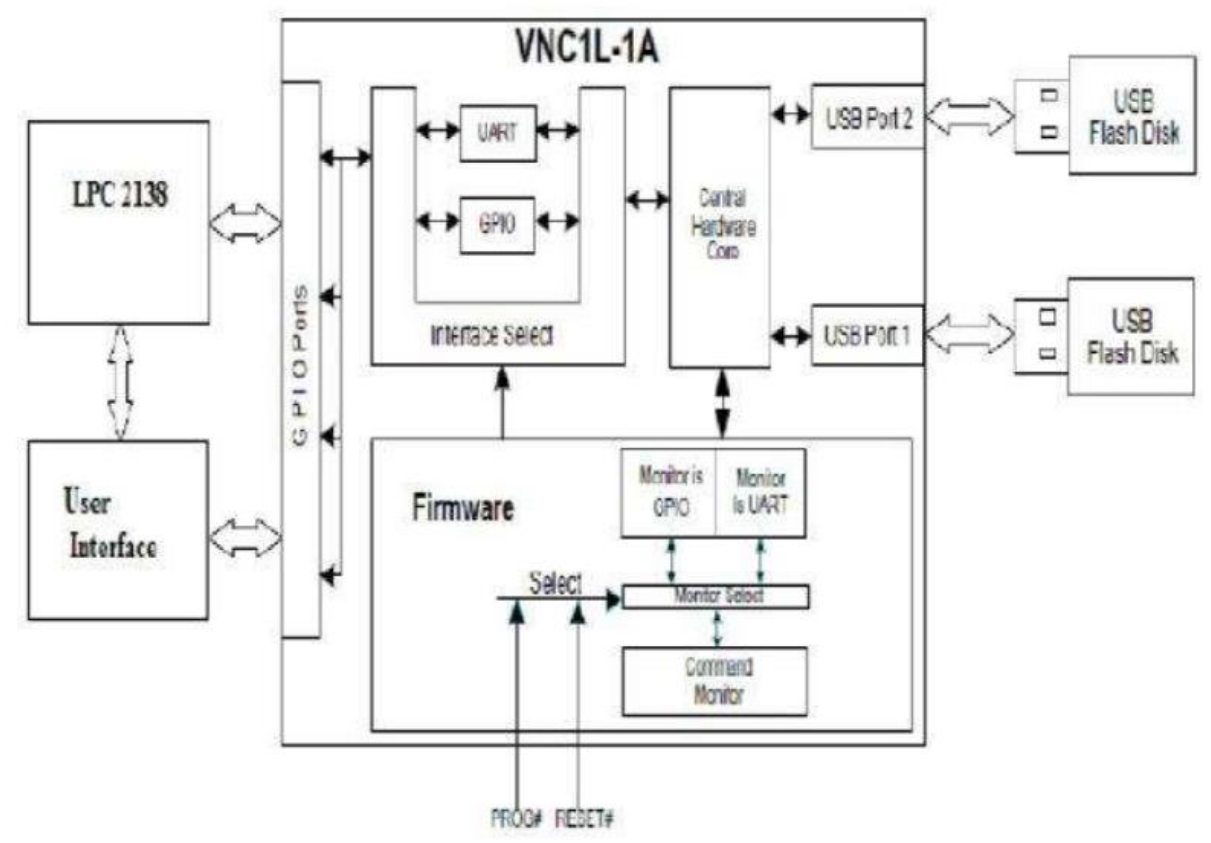




\section{System Flow Chart:}

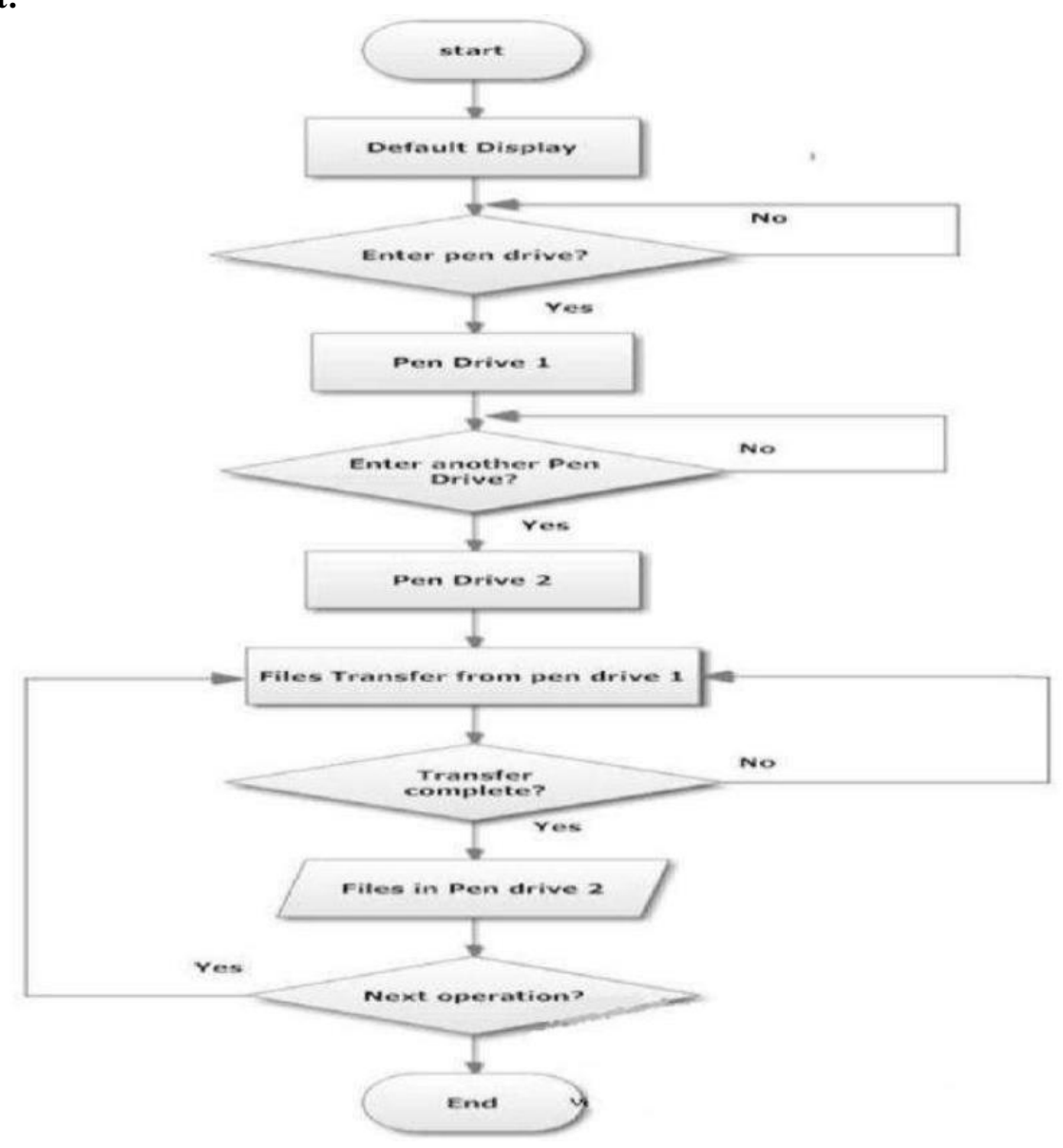

\section{Result:}

In doing our work we always face some problems in our path, problems an success both come in our path. When we worked on the hardware of the project we initially had opted for an ARM board combined with the general purpose board. The idea was taken because we were new with the use of the VNC11 IC. We taken the general purpose board so that we can easily demount the components if something goes wrong. Also it was used so that future modifications can be made easier if required or necessary. We initially mounted all the circuit components related to the ARM board and so far the work was going as we had planned. The problem arised as we mounted the VNCL1 IC, the board became bulky and it became difficult for us to mount all the components that were to be interfaced between ARM and VNCL1. Size of the board became the reason behind the bulkiness. We had to compromise with the spacing of the components and that made the work very untidy and difficult for us to distinguish between various components. So finally we decided to switch the boards.

\section{Conclusion and Future scope:}

we usually used to transfer data from pc to laptop or pc to mobile. It is not so easy task to carry laptops or pc everywhere so we need to design a usb or pendrive onsisting bluetooth due to this sharing data between two USB can be so easy because it is easy to cary. so it will so effective system in future.

\section{References:}

[1].Parmar, C.D.; Dwivedi, V.V.; Wandra, H. H., "Firmware for file transfer between Bluetooth module and flash memory through microcontroller in an emerging concept of wireless portable memory access," Communication Control and Computing Technologies (ICCCCT), 2010 IEEE International Conference on, vol., no., pp.42,46, 7-9 Oct.2010 doi: 10.1109/ICCCCT.2010.5670781

[2].International Journal of Engineering Research and Development, ISSN: 2278-067X, Volume 1, Issue 6 (June 2012), PP. 25-34.

[3].Arfwedson, Henrik and Sneddon, Rob, “Ericss0n's Bluetooth Modules”, Nov 4,1999. Ericsson Review No.4, PP 198205

[4].Robert L. Boylstad, "Electronic Devices and Circuit Theory", Edition Eight.

[5].Behrouz A Forouzan, "Data Communication And Networks", 4th edition.

[6].http://en.wikipedia.org/wiki/USB_flash_drive

[7].http://en.wikipedia.org/wiki/Bluetooth

[8].http://www.bluetooth.org/

[9].http://compnetworking.about.com 
[10]. https://play.google.com/store/apps/details?id=it.medieval.blueftp

[11]. ARM Assembly Language: Fundamentals and Techniques by William Hohl.

[12]. ARM system developers guide by Andrew N.Sloss.

[13]. Embedded systems by Raj kamal

[14]. ARM Architecture Reference Manual by David Seal.

[15]. ARM Assembly Language: Fundamentals and Techniques by William Hohl.

[16]. ARM Microcontroller Interfacing: Hardware and Software by Warwick A. Smith.

[17]. Microprocessors, pc hardware and interfacing by N.Mathivanan. 